\title{
Hybrid Data Fusion and Cooperative Schemes for Wireless Positioning
}

\author{
S. Sand and C. Mensing \\ German Aerospace Center \\ Oberpfaffenhofen, \\ 82234 Wessling, Germany \\ E-mail: stephan.sand@dlr.de
}

\author{
Y. Ma and R. Tafazolli \\ University of Surrey \\ Guildford, Surrey GU2 7XH, \\ United Kingdom \\ E-mail: y.ma@surrey.ac.uk
}

\author{
X. Yin, J. Figueiras, \\ J. Nielsen, and B. H. Fleury \\ Aalborg University \\ DK-9220 Aalborg, Denmark \\ E-mail: xuefeng@es.aau.dk
}

\begin{abstract}
The Wireless Hybrid Enhanced Mobile Radio Estimators (WHERE) consortium researches radio positioning techniques to improve various aspects of communications systems. In order to provide the benefits of position information available to communications systems, hybrid data fusion (HDF) techniques estimate reliable position information. Within this paper, we first present the scenarios and radio technologies evaluated by the WHERE consortium for wireless positioning. We compare conventional HDF approaches with two novel approaches developed within the framework of WHERE. Yet, HDF may still provide insufficient localization accuracy and reliability. Hence, we will research and develop new cooperative positioning algorithms, which exploit the available communications links among mobile terminals of heterogeneous wireless networks, to further enhance the positioning accuracy and reliability.
\end{abstract}

\section{INTRODUCTION}

The main objective of the Wireless Hybrid Enhanced Mobile Radio Estimators (WHERE) project is to study radio positioning techniques using existing and future heterogeneous communications systems and to optimize the various layers of the communication systems (modulation, channel estimation and equalization, radio resource management, etc.) by exploiting the availability of reliable estimator for the position of mobile terminals (MTs) [1]. In order to provide reliable and accurate position information, the WHERE consortium researches hybrid and cooperative positioning.

For reliable positioning, it is necessary to exploit as much positioning information as possible. Typical measurements to compute the position of MTs include time of arrival (TOA), time-difference of arrival (TDOA), angle of arrival (AOA), received signal strength (RSS), Doppler frequency, fingerprinting, etc. Different systems may provide these position based measurements, e.g., cellular mobile radio communications systems, short range communication systems, or even global navigation satellite systems (GNSS's). Potentially each additional available measurement can improve the accuracy, availability, and reliability of the overall position solution.

In a first step, the WHERE consortium studies hybrid data fusion (HDF) algorithms to combine various measurements resulting in a single position solution. Additionally, we will improve the position solution by tracking the position of the MT, e.g., in point-to-point navigation. Yet, data fusion and tracking may still provide insufficient accuracy and reliability.
Thus, in a second step, the WHERE consortium researches cooperative positioning to further enhance the accuracy and reliability of positioning.

\section{SCENARIOS}

As a first task, the WHERE consortium has been discussing and evaluating different scenarios to research and investigate HDF and cooperative positioning algorithms. For some situations, highly accurate positioning solutions are available already today. For instance, the positioning accuracy achieved by using the GNSS systems is in the range of a few meters in rural areas where several satellites can be seen line-of-sight (LOS) [2]. So the focus should be on more challenging environments and scenarios. Finally, we agreed on the following three different scenarios [3].

The first scenario $\mathrm{T} 1$ addresses small-scale situations mainly in indoor environments where short-range ultra-wideband (UWB) links locally complement cellular systems such as the 3rd Generation Partnership Project Long Term Evolution (3GPP-LTE). Alternatively, we may consider a Wi-Fi system cooperating with wireless personal area networks (WPANs), e.g., ZigBee.

The second scenario T2 is a medium-scale indoor scenario, typically in an office-building. Here, a Wi-Fi system complements cellular 3GPP-LTE or cooperates with Worldwide Interoperability for Microwave Access (WiMAX) systems. The indoor scenarios $\mathrm{T} 1$ and $\mathrm{T} 2$ have both in common that there is no GNSS available.

The third scenario T3 is a large-scale environment that considers GNSS and cellular communication system-based positioning in urban canyon situations. Due to the availability of GNSS, the scenario T3 serves as a reference to compare the novel positioning methods developed in WHERE to already available approaches (cf. [4]). In all three scenarios, we assume pedestrian users.

Clearly, the investigations for all scenarios can be extended to several cooperating users where the focus is both on the achievable overall positioning accuracy and the impact on the communications infrastructure. The overall investigation of different scenarios allows to evaluate the performance for a seamless outdoor/indoor navigation using current and future systems. 


\section{HYBRID DATA FUSION}

\section{A. Conventional Hybrid Data Fusion Algorithms}

HDF algorithms in the context of positioning applications estimate the position (if desired also velocity and orientation) of the MT over time. Due to the different kinds of sensors that can provide location-dependent parameters (TOA, TDOA, $\mathrm{AOA}$, etc.), it is a challenging part of the positioning process to combine and weight all measurements in an optimum way [5]. Furthermore, the dependence between the measurements and the position is usually non-linear. Clearly, the navigation equation [5] can be solved on a snap-shot base assuming a static user. A better approach is to use the underlying mobility model of the user as input to the HDF algorithms. It allows to include a-priori knowledge for the current estimate based on previous estimates. For instance, the a-priori knowledge could be a maximum velocity of $10 \mathrm{~km} / \mathrm{h}$ if we assume pedestrian users as very simple mobility model.

Bayesian filter approaches are widely used for HDF and tracking purposes. However, the classical linear Kalman filter (KF) [6] is not directly applicable to this problem due to the non-linear relation between the measurements and the position. Hence, extended KF (EKF) or unscented KF (UKF) are good candidates for such approaches. For instance, the EKF linearizes the measurement and mobility models around a certain working point (based on recent estimates) and applies then the KF to this linearized system. Also more complex particle filters $(\mathrm{PF})$ have gained a lot of attraction recently [7].

Figure 1 shows the cumulative distribution function (CDF) of the position estimation error using a 3GPP-LTE system in an indoor environment as in Scenario T2 [4]. For the simulations, a 3GPP-LTE cellular network with inter-site distance of $1500 \mathrm{~m}$ was assumed and TDOA measurements were extracted from the incoming signals. The navigation equation was solved assuming a static as well as a dynamic user. In the latter case pedestrian users were assumed whose positions are tracked by measures of an EKF approach. The building was assumed to be at the cell edge which is a reasonably well-behaved scenario since signals from several base stations can be received very well. Even though, we see that only in $55 \%$ of the cases the error is below $100 \mathrm{~m}$ for the static solution. With EKF tracking this can be improved to $82 \%$. However, it is obvious that in this critical situation support in terms of additional sensors or cooperative positioning is required to allow a satisfying navigation.

\section{B. Novel Hybrid Data Fusion Algorithms}

Two novel hybrid data fusion algorithms [8], [9] have been proposed recently within the framework of WHERE.

In the first novel approach [8], a particle filter (PF) is used to track the path parameters directly from the received signals. The data fusion that is performed usually as a separate stage in the conventional tracking algorithms, is now implicitly done during the parameter estimation. Simulations show that by exploiting the temporal evolution of the parameters, this

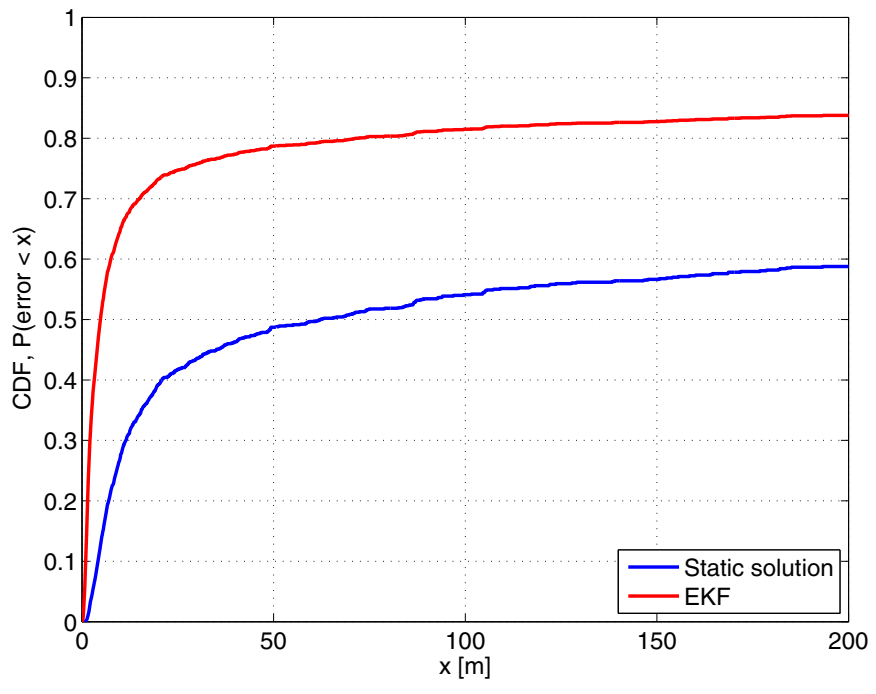

Fig. 1. CDF of the error in indoor position estimation with 3GPP-LTE

novel approach exhibits higher estimation accuracy and robustness against noise and interference than the conventional algorithms.

The second novel approach [9] proposed by WHERE utilizes a single-stage scheme, where the trajectories of object positions in a Cartesian coordinate system are estimated directly from the received signals without introducing any intermediate parameters, such as delays, Doppler frequencies and azimuths of arrival. In this approach, the motion of the object is parameterized by its position, velocity and acceleration. Signals observed from multiple sensors are modeled directly as functions of the object trajectory. The trajectory estimates are updated recursively using a PF. Similar with the first approach, fusion of the observations from multiple sensors is implicitly included when estimating the object trajectories.

Figures 2 (a) and (b) depict the a-posterior pdf of the object trajectory for two examples in a single-object scenario. In these simulations, a multistatic passive radar system is considered, where Digital-Video-Broadcasting Terrestrial (DVB-T) signals reflected by a moving object are obtained with three receivers. The a-posterior pdf exhibits a global maximum that is located at the true object position. This indicates that the trajectory of an object can be estimated by maximizing the a-posterior pdf.

Figure 3 depicts the estimated trajectories obtained by using the proposed PF-based single-stage approach and two conventional tracking algorithms in a single-object scenario. The two latter algorithms use a PF and an EKF respectively to track the object trajectory from the estimates of Doppler frequency and delay of the received signal. It can be observed that the trajectory estimated with the single-stage PF fits the true trajectory well, while the trajectory estimates obtained with the conventional algorithms using multiple stages exhibit significant deviations. Figure 4 depicts the root mean square estimation error (RMSEE) of target position obtained using the proposed single-stage PF and the convectional PF- and EKF- 

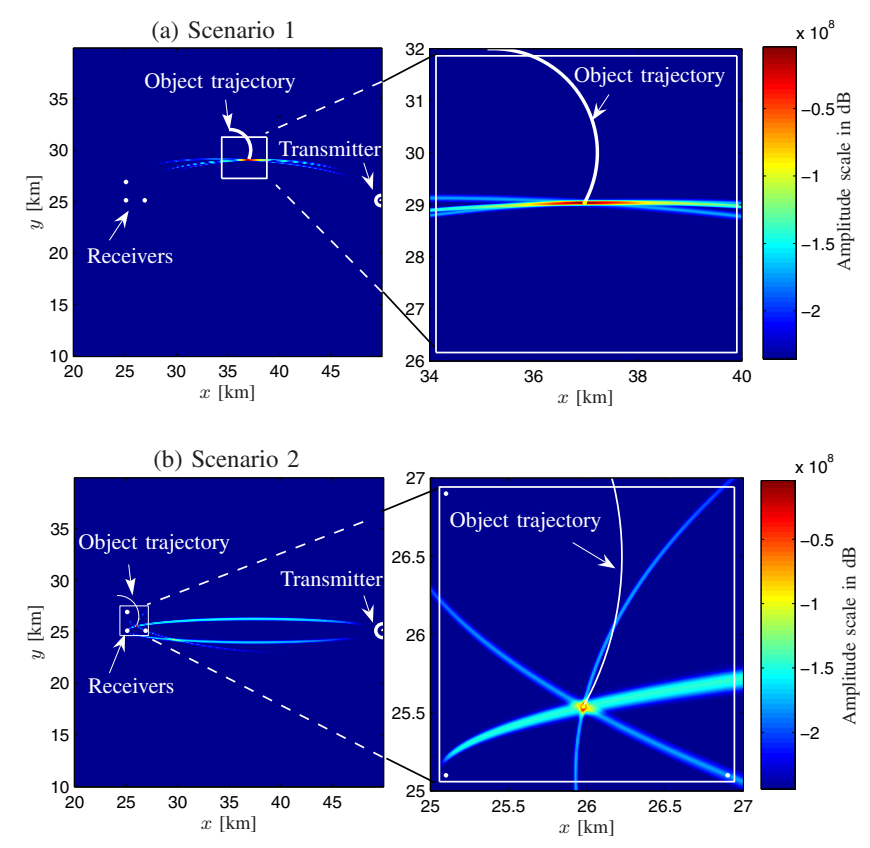

Fig. 2. A-posterior pdf of the object trajectory in a single-object scenario at one observation instant.

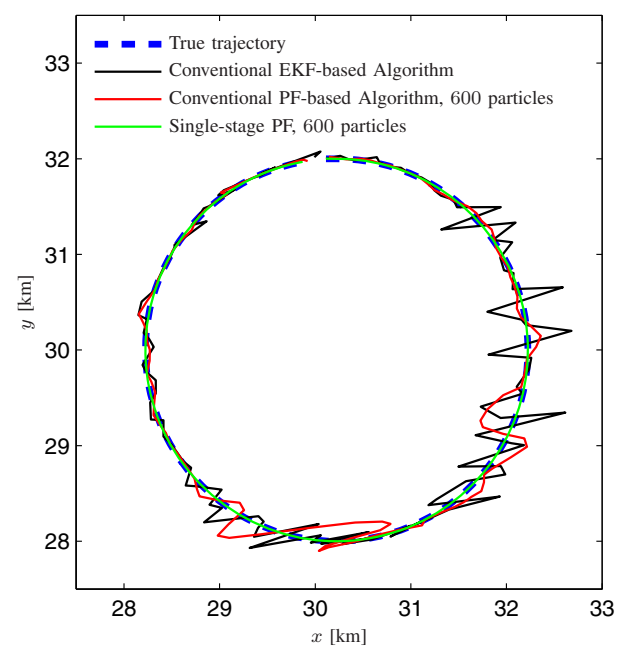

Fig. 3. Estimated object trajectories using different tracking algorithms in a single-object scenario.

based methods in a single-target scenario. The single-stage PF returns RMSEEs less than $20 \mathrm{~m}$ for low SNR, e.g. equal to $-10 \mathrm{~dB}$, and less than $1 \mathrm{~m}$ for SNRs higher than $10 \mathrm{~dB}$. The two conventional algorithms exhibit estimation errors at least one order of magnitude higher than the proposed PF. The novel approach can be easily generalized to the hybrid positioning scenario where observations of an object are available from multiple nodes of an heterogeneous network.

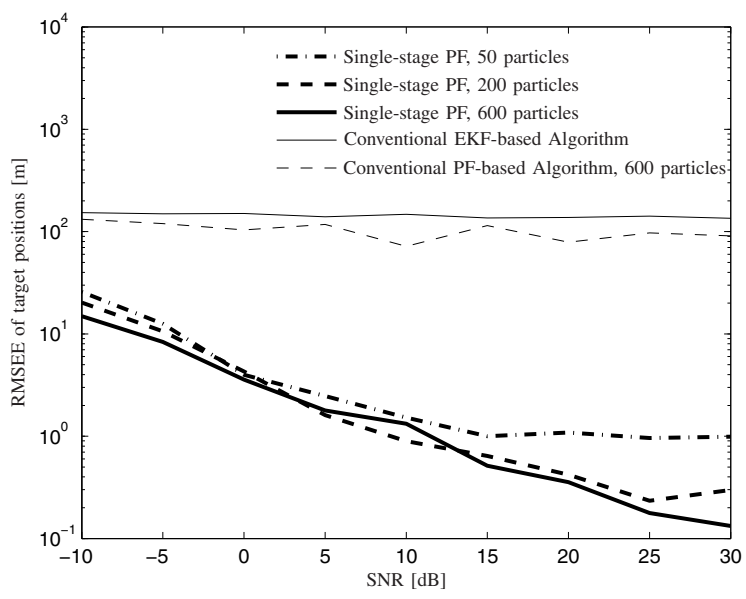

Fig. 4. RMSEE of target positions versus SNR.

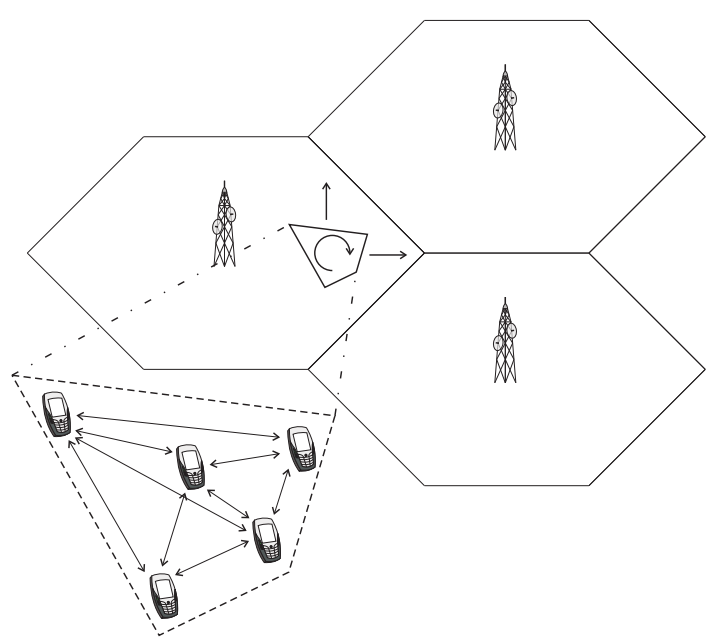

Fig. 5. Schematic representation of the proposed algorithm. Note that the arrows represent estimated variables.

\section{Cooperative Positioning}

Cooperative positioning is a recent research topic in wireless communications systems. These positioning schemes are suitable for scenarios where several technologies and mobile nodes coexist in an heterogeneous network. Figure 5 shows a schematic representation of the concept of cooperative positioning.The goal is to enhance position estimators of the mobile nodes, given that they share link information with nearby nodes. This concept permits the use of spacial diversity in positioning algorithms.

\section{A. Conventional Cooperative Positioning Algorithms}

The concept of cooperative positioning was first proposed in Japan for acquiring real-time positioning information of mobile robots [10]. This concept, mostly applied nowadays to wireless sensor networks (WSN), has been recently introduced to heterogeneous communication networks [11]-[14]. Some of the aspects present in the WSN context are also present in general communication networks, however the heterogeneity of nowadays wireless communication networks can be seen as 
an additional problem to be addressed. Current research has aimed at porting WSN positioning algorithms into communication networks. In [12], the author exploits user cooperation in a least squares framework where cellular and ad-hoc links are combined in a single module of the system. Instead in [11], [13], common Bayesian filtering, namely KF, is used for combining short- and long-range links. In [14] the authors propose a mathematical formulation based on the absolute position obtained by the cellular system followed by a routine of optimization that uses the information from the short-range links. Common aspects and subsequently similar results can be expected for example from Cramér-Rao bound analysis in WSN. The Cramér-Rao bound was analyzed for some example networks [15]. In general, some of the important aspects concerning cooperative positioning in wireless networks are for instance: technology integration in a heterogeneous network, positioning information overhead, distributed computing, and node clustering management. Though multihop positioning, as it is known in the WSN context, is also possible in heterogeneous networks, its implementation may require more robust clustering algorithms since wireless nodes are, by nature, mobile. Current cooperative schemes classify the nodes into two groups:

- "anchor nodes" whose positions are known (e.g., obtained via GNSS or manually set by the operator) and commonly static;

- "unknown nodes" that are located at unknown positions, generally localized with cooperative schemes by sharing of information.

\section{B. Novel Cooperative Positioning Algorithms}

Cooperative positioning techniques can be enhanced at least in the following three aspects:

1) State-of-the-art approaches are based on the assumption that each node has direct connection with the others in LOS. However, in practical wireless networks there are many new nodes that cannot reach anchor nodes or other new nodes due to various factors, such as largescale fades, shadowing effects, limited radiation power, etc. In such cases the current approaches that rely on the assumption of direct LOS perform poorly. Therefore, establishing an appropriate and meaningful mathematical model has a wide implication for cooperative positioning.

2) Signal-processing complexity and training/signaling overhead are two key problems for existing cooperative positioning schemes. For example a network accommodating $N$ nodes, each new node needs to perform distance estimation (range measurement) for $(N-1)$ point-to-point links. This requires significant signal-processing pay particularly for a large $N$. Moreover, each new node needs to broadcast both training information and its measurement results. This can result in significant training/signaling overhead and create difficulties to medium-access control (MAC). In order to resolve these problems, the WHERE consortium will investigate different solutions for balancing the trade-off between signaling/training overhead

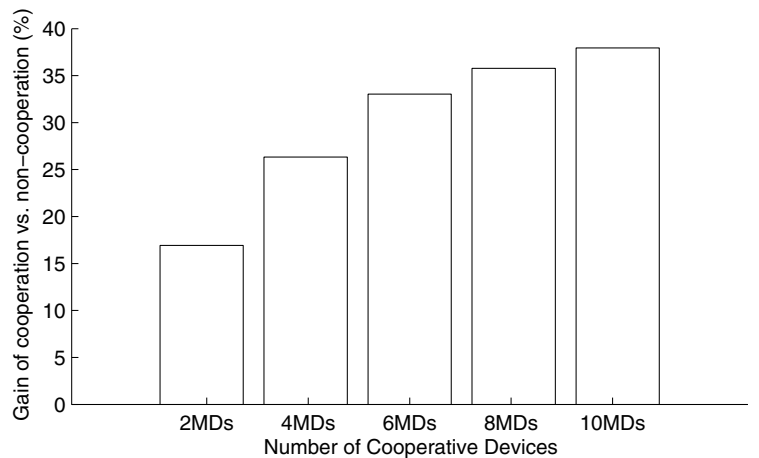

Fig. 6. Gain in accuracy for Mobile cooperative schemes with respect to traditional non cooperative schemes.

and positioning accuracy. This overhead can be reduced e.g. by lowering the measurement/signaling frequency or by limiting the number of cooperating nodes. As shown for a specific scenario in [13], and reproduced in Fig. 6 the positioning accuracy gain that can be obtained when increasing the number of cooperating nodes becomes limited for large node sets. In the considered scenario the accuracy gain when going from 6 to 8 cooperating nodes is less than $5 \%$, whereas the step from 2 to 4 nodes increases the accuracy by approximately $25 \%$. Besides the number of cooperating nodes, the locations of the cooperating nodes will have a significant influence on the accuracy. For example, we observe on the right hand side of Figure 2 that the maximum of the location pdf is either diffuse or sharply concentrated dependent on the locations of the sensors. This motivates a proper selection and partitioning of cooperating nodes. Specifically the WHERE consortium proposes to perform cooperative positioning for a group of nodes. Node grouping and selection strategies should be carefully devised in terms of factors such as group size, estimation accuracy, etc.

3) Lacking LOS to anchor nodes is one of the major motivations for the WHERE project to consider cooperative positioning. Therefore, one of the key questions, the WHERE project seeks to address is: what is the minimum number of anchor nodes that is required for offering sufficient accurate positioning information? The CramérRao bound is a good metric to analyze this issue and shows the impact of the constellation of anchor nodes on the positioning accuracy. The WHERE consortium will first investigate this problem for some special examples with the assumption of perfect synchronization, and then extend results to more general cases.

\section{Conclusions}

To conclude, the WHERE consortium researches novel HDF and cooperative schemes for wireless positioning to provide accurate and reliable location information. Among others, we focus on short-range UWB and WPAN, mid-range Wi-Fi, and long-range 3GPP-LTE and WiMAX radio technologies for 
positioning besides GNSS.

In a conventional two-step HDF algorithm, the position dependent parameters are first computed from individual snapshots of the received signal. Then, the HDF algorithm tracks the position through these parameters. In contrast, we present two novel PF based HDF algorithms, which either track the path dependent parameters or the position directly from the received signal.

Since in some cases, the position accuracy and reliability may still not be sufficient to improve communications systems, we research new cooperative positioning schemes. Here, the focus of our work will be on establishing appropriate mathematical models accounting for LOS and non-LOS propagation, balancing the trade-off between signaling overhead and positioning accuracy, and finding the minimum number of required anchor nodes for offering sufficiently accurate positioning information.

With the accurate and reliable position information obtained by HDF and cooperative schemes, we can then enhance the performance of mobile communication systems.

\section{ACKNOWLEDGMENT}

This work has been performed in the framework of the ICT project ICT-217033 WHERE, which is partly funded by the European Union.

\section{REFERENCES}

[1] "EU-ICT FP7 Project Wireless Hybrid Enhanced Mobile Radio Estimators (WHERE)," http://www.ict-where.eu/.

[2] P. Misra and P. Enge, Global Positioning System: Signals, Measurements and Performance. Ganga-Jamuna Press, 2004.
[3] Definition of the WHERE framework and scenarios, EU ICT-217033 Wireless Hybrid Enhanced Mobile Radio Estimators (WHERE) Project, Mar. 2008, D1.1, Version 1.0.

[4] "GSA FP6 Project Galileo Receivers for Mass Market (GREAT)," http://www.greatproject.org/.

[5] A. H. Sayed, A. Tarighat, and N. Khajehnouri, "Network-Based Wireless Location," IEEE Signal Processing Magazine, vol. 22, no. 4, pp. 24-40, July 2005.

[6] S. M. Kay, Fundamentals of Statistical Signal Processing: Estimation Theory. Prentice Hall, 1993.

[7] M. S. Arulampalam, S. Maskell, N. Gordon, and T. Clapp, "A Tutorial on Particle Filters for Online Nonlinear/Non-Gaussian Bayesian Tracking," IEEE Transactions on Signal Processing, vol. 50, no. 2, February 2002.

[8] X. Yin, T. Pedersen, G. Steinböck, G. E. Kirkelund, P. Blattnig, A. Jaquier, and B. Fleury, "Tracking multi-dimensional time-variant parameters of radio propagation paths using particle filtering," Proceedings of IEEE Radar Conference (RadarCon), May 2008.

[9] X. Yin, T. Pedersen, P. Blattnig, A. Jaquier, and B. Fleury, "A Single-Stage Target Tracking Algorithm for Multistatic DVB-T Passive Radar Systems," Submitted to IEEE Global Communications Conference (Globecom), Nov. 2008.

[10] R. Kurazume, S. Hirose, S. Nagata, and N. Sashida, "Study on cooperative positioning system (basic principle and measurement experiment)," in IEEE ICRA, Minnesota, USA, 1996.

[11] S. Frattasi and J. Figueiras, Ad-Coop Positioning System : Using an Embedded Kalman Filter Data Fusion. $\quad$ CRC Press, 2007, pp. 120 131.

[12] S. Frattasi, "Link layer techniques enabling cooperation in fourth generation (4g) wireless networks," Ph.D. dissertation, Aalborg University, September 2007.

[13] J. Figueiras, "Accuracy enhancements for positioning of mobile devices in wireless communication networks," Ph.D. dissertation, Aalborg University, January 2008.

[14] Q. Cui, J. Liu, X. Tao, and P. Zhang, "A Novel Location Model for 4G Mobile Communication Networks," 2007, pp. 274-278.

[15] E. G. Larsson, "Cramér-Rao bound analysis of distributed positioning in sensor networks," in IEEE Signal Processing Letts, 2004. 